\title{
GLAD!
}

Revue sur le langage, le genre, les sexualités

$03 \mid 2017$

Hétérosexualités

\section{Devenir post-straight?}

Auto-ethnographie d'un artiste-chercheur hétérosexuel

Becoming Post-Straight? Auto-Ethnography of a Heterosexual Artist Scholar

\section{Luc Schicharin}

\section{(2) OpenEdition}

Journals

Édition électronique

URL : http://journals.openedition.org/glad/908

DOI : $10.4000 /$ glad.908

ISSN : 2551-0819

Éditeur

Association GSL

\section{Référence électronique}

Luc Schicharin, « Devenir post-straight ? », GLAD! [En ligne], 03 | 2017, mis en ligne le 10 décembre 2017, consulté le 20 janvier 2021. URL : http://journals.openedition.org/glad/908 ; DOI : https:// doi.org/10.4000/glad.908

Ce document a été généré automatiquement le 20 janvier 2021.

\section{(c)}

La revue GLAD! est mise à disposition selon les termes de la Licence Creative Commons Attribution -

Pas d'Utilisation Commerciale - Pas de Modification 4.0 International. 


\title{
Devenir post-straight?
}

\author{
Auto-ethnographie d'un artiste-chercheur hétérosexuel \\ Becoming Post-Straight? Auto-Ethnography of a Heterosexual Artist Scholar
}

\section{Luc Schicharin}

Cette exploration inclut au fil du texte une discussion entre le comité de rédaction et l'auteur sous forme de questions/réponses. Nous avons souhaité restituer, dans la version finale du texte, cette dimension dialogale qui apparaît dans le texte sous la forme d'un jeu de questions-réponses. Lae lecteur.e pourra ainsi choisir à son gré de lire le texte original (en noir), puis les discussions que celui-ci a soulevées entre l'auteur et les éditrices, ou bien opter pour une lecture linéaire, avec les irruptions polyphoniques qu'elle implique. Ces interruptions, croyons-nous, ouvrent une fenêtre sur les coulisses d'un texte, pour le révéler comme une élaboration, et participent ainsi à en faire un objet en mouvement, un dialogue en cours.

1 À la fin des années 1970, Monique Wittig avait défini la pensée straight ${ }^{1}$ comme une oppression matérielle par le langage hétérosexuel. Selon la théoricienne, le pouvoir straight est une instance omniprésente qui exerce sa violence par une oppression intellectuelle, verbale et physique de toute contestation et de toute alternative à l'hétérosexualité. Ce potentiel contestataire et alternatif provient des sujets minoritaires - et Wittig insiste davantage sur les lesbiennes et les hommes homosexuels, bien qu'elle parle aussi des femmes, des Noirs et des esclaves - qui adoptent un langage corporel et sexuel qui n'appartient pas au lexique scientifique, social et politique de la pensée straight. Ainsi, si la pensée straight combat violemment les contradictions qui s'en prennent à son régime, c'est parce qu'elle veut sauvegarder le privilège social de l'idéologie hétérosexuelle dominante dans laquelle tout (discours) ne devrait être qu'hétérosexuel ou, en tous les cas, se rapporter d'une manière ou d'une autre à l'hétérosexualité blanche qui est considérée comme universelle. Wittig spécifie ainsi :

Bien qu'on ait admis ces dernières années qu'il n'y a pas de nature, que tout est culture, il reste au sein de cette culture un noyau de nature qui résiste à l'examen, 
une relation qui revêt un caractère d'inéluctabilité dans la culture comme dans la nature, c'est la relation hétérosexuelle ou relation obligatoire entre «l'homme » et «la femme». Ayant posé comme un principe évident, comme une donnée antérieure à toute science, l'inéluctabilité de cette relation, la pensée straight se livre à une interprétation totalisante à la fois de l'histoire, de la réalité sociale, de la culture et des sociétés, du langage et de tous les phénomènes subjectifs. Je ne peux que souligner ici le caractère oppressif que revêt la pensée straight dans sa tendance à immédiatement universaliser sa production de concepts, à former des lois générales qui valent pour toutes les sociétés, toutes les époques, tous les individus. $^{2}$

2 La pensée straight est une conquête de la pensée collective par le langage, c'est une injonction politique, culturelle et scientifique à l'hétérosexualité; elle s'adresse à l'ensemble des sujets (à travers le monde et à travers le temps) et, plus particulièrement, à ceux et celles qui ne répondent pas à cette assignation hétérosexuelle; c'est aussi une manière, pour les tenants de la parole, de poser un discours surplombant qui ne tient pas même compte des discours contestataires formulés par les subjectivités qui sortent du cadre sémiologique de l'hétérosexualité. Par avance, à travers l'inoculation philosophique de concepts scientifiques prétendus essentialistes, diffusés dans la littérature académique et les médias dans leur ensemble, la pensée straight tente d'éradiquer toute tentative individuelle et groupusculaire de contradictions à l'encontre de son régime intellectuel, social et politique. Et puisque nous évoquons le langage, n'ayons pas peur des mots, il s'agit bien là d'une forme de propagande institutionnalisée et imposée à la population par un ensemble d'acteurs influents ${ }^{3}$ (quel que soit leur domaine d'activité) dans la construction de la vie sociale sur un territoire donné - et qui aspire à une extension fulgurante. L'entreprise sémiologique et politique de la pensée straight consiste ainsi à faire taire ou à rendre inaudibles les subjectivités minoritaires.

Dans le contexte plus particulier de la société française actuelle, les derniers bouleversements sociaux autour de la loi sur le «mariage pour tous $»^{4}$ (17 mai 2013) nous amènent à redéfinir la pensée straight aujourd'hui. En effet, l'adoption de ce texte de loi a soulevé de vives protestations: la droite conservatrice voit le mariage homosexuel comme une stratégie politique visant à imposer la «théorie du genre » et la déconstruction de l'hétéro-normativité. Et les militant.e.s et intellectuel.le.s queer ${ }^{5}$ perçoivent le mariage homosexuel comme une tentative de contrôler les minorités sexuelles au sein de pratiques culturelles et sociales normatives inspirées de l'hétérosexualité. Alors le mariage homosexuel constitue-t-il une défaite ou une simple mise à jour de la pensée straight ? Tout porte à croire qu'il s'agit d'une négociation du pouvoir avec les contre-pouvoirs, accordant aux homosexuel.le.s un droit à une existence publique, dans les registres de l'État, mais uniquement dans un cadre culturel et politique - l'institution du mariage - qui est défini, sur le plan légal, et contrôlé par le régime hétérosexuel de la société, c'est-à-dire par la pensée straight. Force est de constater que le langage dominant de la législation dissimule en réalité une main de fer dans un gant de velours : la violence philosophique de la contrainte législative à un style de vie hétérosexuelle par la création d'un "mariage homosexuel » est masquée derrière les apparences d'une reconnaissance officielle de l'homosexualité par la pensée straight ${ }^{6}$.

4 En outre, Wittig insiste sur le fait que la pensée straight - dont la terminologie peut tromper les lecteurs.trices - n'opprime pas uniquement les minorités sexuelles. Ainsi précise-t-elle : 
La société hétérosexuelle n'est pas la société qui opprime seulement les lesbiennes et les hommes homosexuels, elle opprime beaucoup d'autres-différents, elle opprime toutes les femmes et de nombreuses catégories d'hommes, tous ceux qui sont dans la situation de dominés. [...] Le concept de "différence des sexes » par exemple constitue ontologiquement les femmes en autres différents. Les hommes eux ne sont pas différents. (Les Blancs non plus d'ailleurs ni les maitres mais les Noirs le sont et les esclaves aussi). ${ }^{7}$

5 Ainsi, la pensée straight est définie par Wittig comme une oppression scientifique et politique (institutionnalisée) visant à rendre hégémonique la situation corporelle et sociale de l'homme blanc hétérosexuel. De ce fait, concevoir une hétérosexualité poststraight exige, pour un contestataire masculin blanc hétérosexuel, de réviser sa posture oppressive sur le plan de la sexualité, bien entendu, mais également sur celui du genre et de la race. C'est pourquoi, si l'on désire comprendre et pouvoir répondre (correctement, complètement et positivement) à la proposition théorique de Wittig, la traduction française du terme «straight " par le mot « hétérosexuel » est insuffisante. En effet, ce qui est désigné par la critique wittigienne n'est pas tant l'hétérosexualité que la pensée dominante, la règle, ce qui est relatif à la loi en quelque sorte. La pensée straight produit un discours homophobe, sexiste et raciste, mais néanmoins fondateur, qui favorise ce que Judith Butler nommera par la suite " l'hégémonie hétérosexuelle ${ }^{8}$ ". Ce discours fait loi, et cette loi peut être désignée comme symbolique, lorsqu'elle n'est pas inscrite dans les textes officiels, mais ses répercussions sociales sur les sujets dominés sont très matérielles et très concrètes - puisqu'elle parvient à ses fins en produisant des paroles ou des actes de violence homophobe, raciste et sexiste. Wittig nous invite à considérer le fait que les notions d' « altérité » et de " différence » sont des euphémismes politiques que les dominants ont inventés pour qualifier une «situation historique de domination ». La théoricienne rappelle que c'est aussi par l'invention de cette catégorie totalisante de la «différence » et de «l'Autre » que la pensée straight cherche à faire dominer ses concepts et son lexique, disqualifiant de fait l'invention d'autres appellations signifiantes susceptibles de perturber les structures normatives de la réalité sociale conçue sur mesure par les dominants. C'est pourquoi, selon Wittig, pour lutter contre le pouvoir linguistique de la pensée straight, il faut cesser d'employer certains mots ou en tout cas cesser de les considérer comme allant de soi :

Pour nous il ne peut plus y avoir de femmes, ni d'hommes, qu'en tant que classes et qu'en tant que catégories de pensée et de langage, ils doivent disparaître politiquement, économiquement, idéologiquement. Si nous lesbiennes, homosexuels nous continuons à nous dire, à nous concevoir des femmes, des hommes, nous contribuons au maintien de l'hétérosexualité. ${ }^{9}$

L'utilisation du terme « lesbienne » apparaît alors à Wittig comme un acte de résistance langagière permettant de sortir du cadre hétérosexuel où - pour le dire brutalement la pensée straight désigne les individus nés avec un vagin comme des femmes et les individus nés avec un pénis comme des hommes. Faut-il répéter que cette violence des assignations de genre est très matérielle puisqu'elle justifie, depuis les années 1950 jusqu'à nos jours, l'usage de la torture médicale à l'encontre des personnes intersexuées qui sont mutilées à la naissance afin de pouvoir répondre à l'injonction politique à la binarité homme/femme - soi-disant ontologique ${ }^{10}$ ? En signe de protestation contre la binarité du genre, nous avons vu avec Butler ${ }^{11}$ que, suivant la perspective wittigienne, les minorités vont faire proliférer les identités sexuelles dans les subcultures queer et trans*, et cela bien au-delà des espérances de Wittig elle-même qui ne proposait d'investir que le mot « lesbienne ». En outre, rappelons également que 
les sujets transidentitaires vont fournir des usages impropres de l'assignation de genre, permettant l'écriture d'un corps et d'une identité qui vient contredire la pensée straight - bien que le design corporel des trans* sous hormones et/ou opérés reste parfois encore, pas toujours, sous le contrôle médicalisé du pouvoir hétérosexuel.

Ces actes de contestation queer, qui utilisent le langage (discursif ou corporel) que l'on pourrait qualifier de non-straight, m'ont inspiré et m'ont incité à penser qu'il est sans doute possible de lutter à partir d'une position d'homme blanc hétérosexuel - qui est la mienne -, bien qu'il s'agisse de l'identité hégémonique par excellence. Cependant, est-il possible, pour un sujet hétérosexuel - qui plus est lorsqu'il cumule d'autres signes de la domination sociale (la masculinité, la blanchité et l'identité cisgenre) -, d'échapper à l'emprise sémiotique et politique de la pensée straight? Autrement dit, les hétérosexuel.le.s sont-ils/elles capables de donner sens à la société par-delà le point de vue dominant de l'hétérosexualité ? La question se pose car, comme l'a dénoncé Monique Wittig, la pensée straight - qui n'est autre qu'une idéologie qui naturalise l'hétérosexualité blanche et instaure des rapports de domination entre les catégories d'individus - s'infiltre dans l'ensemble des structures sociales qui régissent la construction identitaire des groupes humains et des individus.

C'est pourquoi je souhaite développer quelques pistes de réflexion à travers un travail d'auto-ethnographie: en tant qu'homme blanc hétérosexuel et universitaire, qui a bénéficié des apports intellectuels conséquents des études minoritaires (féministes et queer $)^{12}$, je peux dire que la théorie esthétique des langages subculturels queer - dans les arts, la littérature, la philosophie, les sciences politiques et sociales - m'a donné les outils adéquats afin de pouvoir critiquer le modèle universaliste de la pensée straight. Ce qui a été l'occasion pour moi d'envisager de nouvelles possibilités d'exister, de penser et de parler en dehors du système hétérosexuel.

GLAD! : L'articulation entre système hétérosexuel et identité hétérosexuelle est un nœud d'achoppement intéressant. La notion de post-straight permetelle de rendre visible cette articulation?

Avec ma proposition de penser une posture hétérosexuelle poststraight, je voudrais réfléchir à une méthode d'action à l'encontre des normes à partir d'une position considérée comme dominante. En étudiant ma propre expérience, je questionne mon «destin biologique » d'homme blanc, hétérosexuel, cisgenre, valide : ce corps me conduit-il systématiquement et irrémédiablement à un «destin politique » qui est l'oppression des minorités ? J'aimerais proposer de répondre par la négative: malgré une identification socialement dominante (ou presque), je renonce à la violence de mon pouvoir à travers la création personnelle et autogérée d'un comportement non oppressif et non agressif. Je cherche aussi à comprendre comment cela est rendu possible. Quels sont les interstices de mon identité qui sont ou ont été socialement dévalués, malgré ma posture dominante en tant qu'homme hétérosexuel blanc? Et comment cela m'a-t-il permis de tisser des connexions politiques avec des problématiques de domination sociale qui ne me concernent pas directement (la race, le genre) ? Comment, à partir de ce vécu passé et présent, m'est venue la nécessité d'envisager mon hétérosexualité différemment? 
J'examinerai plus en détail ce que cela signifie et ce qu'implique le fait pour un homme hétérosexuel blanc de déconstruire la pensée straight. Mais avant cela, je voudrais attirer l'attention sur une question préalable : comment devient-il possible d'accorder du crédit et de l'importance aux discours féministes, afroféministes, crip et queer, alors même qu'ils s'opposent à la pensée straight qui me place en principe dans une situation sociale dominante, censée empêcher toute remise en question du système qui me privilégie $^{13}$ ? Pour reformuler ces questions à travers une métaphore cinématographique qui se réfère à la fois aux écrits de Judith Butler (avec Trouble dans le genre $^{14}$ ) et à la filmographie des sœurs Wachowski (avec Matrix $\left.{ }^{15}\right):$ comment ai-je choisi la pilule queer plutôt que la pilule straight, la critique queer plutôt que l'apologie de la «matrice hétérosexuelle»? En ce qui concerne mon propre engagement, cela est en partie le résultat d'une formation scolaire et universitaire à la pensée critique, mais c'est aussi la conséquence du développement antérieur d'un esprit critique ou d'une attitude critique préalable. Jacques Boisvert affirme que, pour aiguiser sa pensée critique, «la personne doit en effet manifester un certain nombre d'attitudes, de dispositions, d'habitudes de pensée et de traits de caractère que l'on peut regrouper sous l'étiquette "attitude critique" ou "esprit critique" ", ajoutant que "de façon générale, cela signifie que le penseur (sic) critique doit non seulement être capable d'évaluer des raisons adéquatement, mais qu'il doit aussi avoir tendance à le faire, $y$ être disposé16 ". Cependant, alors que Boisvert écrit que cet esprit critique s'enseigne et s'acquiert à l'école, par le biais de l'enseignement - ce qui apparait problématique à bien des égards ${ }^{17}-$, nous postulons qu'il se construit aussi et surtout à travers une expérience personnelle, en interaction permanente avec la famille, la société et la culture. J'insiste sur la répercussion qu'a pu avoir mon vécu social sur la construction de mon esprit critique ${ }^{18}$. J'écrivais à l'instant que, dans mon cas, le développement d'un esprit critique en prise avec les injustices sociales est antérieur à la formation universitaire, et, en disant cela, il ne s'agit évidemment pas de retirer au féminisme, à l'afroféminisme, à la théorie queer et à la théorie crip le mérite d'avoir transformé - et même révolutionné - mon rapport politique à la société. Cependant, je voudrais tracer un itinéraire, une épistémologie de ma propre pensée critique, et ainsi souligner les imperfections de l'assujettissement par le pouvoir straight. Pour cela, il me faut développer quelques passages autobiographiques, c'est une parenthèse essentielle si l'on veut comprendre comment la subjectivité dominante à laquelle on m'assimile a pu échapper - en partie au moins - à la fonction politique de vecteur philosophique d'une hégémonie culturelle masculine, blanche, hétérosexuelle, valide...

10 Je suis issu de la classe ouvrière blanche, né d'un père mineur aux Houillères du bassin de Lorraine et d'une mère au foyer souffrant à la fois d'une maladie chronique de longue durée et d'un handicap moteur - qui l'oblige à se déplacer en fauteuil roulant à l'extérieur de son logement. Ma scolarité et mon adolescence se sont déroulées dans une zone urbaine dite «sensible» en Lorraine, du fait de la présence d'une petite délinquance très active (trafic de stupéfiants, dégradation du mobilier urbain). Mon quartier était cependant riche d'une forte population immigrée et multiculturelle (principalement maghrébine et turque). De par mon profil de fils de mineur, j'ai expérimenté les inégalités sociales vécues par la classe ouvrière blanche - la méfiance des plus aisés, refusant que leurs enfants m'invitent chez eux, parce que nos parents n'étaient pas du même milieu socioprofessionnel. La grande majorité de mes ami.e.s proches étaient des fils et filles d'immigré.e.s, et c'est donc indirectement que j'ai 
parfois eu l'occasion de constater la violence raciste des institutions - la déchéance sociale à l'école et les contrôles policiers plus appuyés de mes camarades à la peau plus foncée. Indirectement encore, j'ai partagé les complications sociales et politiques concrètes qu'a entraîné le handicap de ma mère - les difficultés à pouvoir se loger et se déplacer dans un espace public qui est avant tout pensé et construit pour les personnes valides. Ces situations critiques vécues à l'adolescence - dont certaines se prolongent jusqu'à aujourd'hui - ont progressivement forgé mon esprit critique et m'ont permis de constater de visu les violences sociales de la pensée straight - lorsqu'elles oppriment les Blancs issus des classes subalternes, les personnes de couleur dans un contexte urbain sensible et les individus handicapés (et notamment les femmes au foyer particulièrement dépendantes et donc vulnérables). Cette sensibilisation par le vécu a engendré un mépris idiosyncratique de toute forme de domination, c'est ainsi que j'ai rallié les luttes politiques contre le sexisme et l'homophobie, ce qui m'a finalement conduit à m'intéresser au féminisme intersectionnel de la théorie queer ${ }^{19}$ auquel j'ai eu accès à l'université (bien conscient que l'acquisition de ce savoir était un privilège).

GLAD! : Ce passage d'un vécu particulier à un cadre général de la critique de la domination est aussi intéressant que singulier. De nombreuses personnes vivent une domination de classe qu'illes placent, en termes de pratiques et d'analyse, au-dessus de la question féministe par exemple (c'est un classique $\mathrm{du}$ discours de la lutte des classes), de la même façon que - dans une proportion moindre - certains discours font primer la race, le genre ou les sexualités sur d'autres rapports sociaux. Or, la question de la sexualité vers laquelle vous vous orientez intellectuellement et dans laquelle vous trouvez des outils, est apparemment l'une des seules que vous ne rencontrez pas dans votre vécu. C'est donc une posture toute particulière que de choisir d'inclure une domination que l'on n'a pas côtoyée pour étendre sa critique à l'ensemble des rapports sociaux, qui va d'une certaine manière à l'encontre d'une politique identitaire. S'agit-il d'un choix ou bien d'une rencontre conceptuelle?

La conscience de la domination de classe a été pour moi une réelle porte d'entrée, bien que cela n'ait pas tout de suite été conscientisé. Bien entendu, il a fallu un parcours complexe, en plusieurs étapes. Mais je voudrais néanmoins évoquer une expérience sociale très personnelle et très marquante qui peut certainement constituer un indice pour mieux expliquer mon ouverture à plusieurs problématiques de dominations sociales: lorsque ma mère a commencé à développer les signes aigus de sa myopathie, mes parents et moi habitions dans un HLM avec plusieurs étages, sans ascenseur, ce qui rendait chacun des déplacements de ma mère à l'extérieur très douloureux. Mon père s'était donc renseigné sur les démarches à entreprendre pour que nous déménagions dans un appartement de plain-pied, mieux adapté au handicap de ma mère. Mais ce type de logement (dans la ville où mon père était affecté) était cependant réservé à des gradés de l'entreprise. Mon père a donc passé des concours pour obtenir le grade requis, mais il était en concurrence avec d'autres personnes ayant des relations au sein de l'entreprise et n'a donc pas obtenu sa promotion, malgré de meilleurs résultats. Cet «échec» a longtemps bloqué le processus de 
déménagement, puis un jour le problème s'est finalement réglé grâce à l'appui de mes professeures de l'école primaire qui avaient pris connaissance de ma situation familiale et qui ont pu nous fournir une aide considérable.

En partageant cette anecdote, je voudrais pointer comment des expériences de vie concrètes, bien qu'indirectes, m'ont permis d'accéder tout à la fois aux questions de genre (le manque de considérations pour les problèmes des femmes de mineur), de classe (les inégalités engendrées par le fait qu'un ouvrier ne soit pas assez gradé), de validisme (l'incapacité de comprendre les spécificités de la vie des handicapé.e.s). Par ce vécu, il m'a été possible d'acquérir la conscience que la domination sociale peut agir de bien des manières, opprimer diverses identités minoritaires, et qu'un seul et même sujet (comme ma mère) pouvait cumuler plusieurs statuts sociaux subalternes. C'est dans ce cadre que j'ai progressivement acquis et produit des outils conceptuels pour élargir la problématique et que, bien plus tard, je me suis mis à penser et vivre les enjeux politiques de mon hétérosexualité masculine.

Réfléchir la domination masculine dans l'espace (hétéro)sexuel est complexe, je me suis un temps tourné vers le post-porno féministe/ queer pour tenter de réinventer mon corps hétérosexuel, ses désirs et ses plaisirs. Cependant, je me suis rendu compte que parfois les modèles critiques et alternatifs du post-porno ayant pour projet de représenter une autre (meilleure ?) sexualité ne correspondent pas à la réalité objective pour un homme ou une femme post-straight: par exemple, tous les couples hétérosexuels conscientisés ne pourront pas fantasmer sur l'inversion des rôles sexuels ou de genre, quand bien même cela a pu apparaître comme la bonne solution pratique (en théorie) pour pallier les inégalités qu'implique la présumée passivité sexuelle des femmes. Comme le suggère Lynda Hart dans La Performance sadomasochiste, les postures de domination/soumission dans le cadre des jeux sexuels (qui sont finalement très proches de la dialectique hétérosexuelle normative passif/actif) sont toujours instables et, parfois, bien que l'on reste "dans son genre", des inversions de pouvoir se produisent, cependant elles ne correspondent pas toujours aux nouvelles représentations sexuelles féministes/queer. Le discours féministe/queer a néanmoins été utile pour ma partenaire et moi (qui sommes en couple depuis presque treize ans) en ce qu'il a facilité les discussions autour des enjeux politiques sous-jacents de notre relation hétérosexuelle; ces échanges nous ont permis d'établir ensemble la signification politique de nos interactions dans l'intimité, et (éventuellement) de resignifier les rapports de pouvoir qu'elles supposent.

11 Ma démarche auto-ethnographique me permet d'affirmer une position singulière dans le régime politique du pouvoir straight, puis dans le féminisme queer, m'offrant un angle particulier pour incorporer les critiques qui me sont adressées en tant qu'homme hétérosexuel blanc et formuler ma propre déconstruction de la pensée dominante. Bien 
entendu, étant donné mon profil sociopolitique, il m'est très difficile de produire une identité corporelle/sexuelle qui vienne contrarier le pouvoir straight, d'autant que je me méfie désormais des appropriations culturelles vers lesquelles je m'étais tourné autrefois ${ }^{20}$ et que je vois aujourd'hui suggérées par certaines féministes lesbiennes médiatiques ${ }^{21}$.

GLAD! : Comme toute appropriation culturelle, cette idée d'une appropriation culturelle du queer est assez problématique, en ce qu'elle crée parfois des paradoxes dans lesquels il faudrait que les hétéros se produisent comme hétéros pour ne pas faire de l'appropriation culturelle. Or, la question est compliquée: le fait que des hétéros adoptent ces traits culturels en légitimant ces codes et en les dépolitisant pose problème, mais est-ce le problème de l'adoption des pratiques culturelles en elles-mêmes? Il est évidemment difficile et risqué de faire le tri. C'est peut-être davantage une question de discours porté, et de sa mise en valeur dans l'espace public.

Lorsque j'évoque le risque d'une appropriation culturelle de la culture queer, il ne s'agit pas pour moi de dicter un code de conduite ou d'éthique pour les «bons hétérosexuels post-straight» mais simplement d'engager une réflexion sur les modalités et les formes que l'on peut donner à une alliance. J'ai adopté par le passé une stratégie corporelle que je pensais efficace à l'époque, et qui consistait à modifier mon apparence physique (me teindre les cheveux en rose, par exemple). Par ce geste, il s'agissait de reproduire certaines des stratégies d'une communauté qui n'était pas vraiment culturellement ni politiquement la mienne. Mais cet engagement corporel ne restait finalement que très superficiel, et n'apportait rien de plus à ce que je pratiquais déjà : une théorie de l'art et des cultures minoritaires. Avec le recul, ces actes corporels «d'apparence», inefficaces sur le plan politique et identitaire, m'apparaissent problématiques, c'est pourquoi je formule cette sorte d'autocritique à rebours. Je me sens bien plus à l'aise aujourd'hui avec l'idée d'offrir mon aptitude à la théorie pour développer une réflexion autour des problématiques queer et tenter de faire avancer la politique des minorités à mon petit niveau (qui est celui de la critique esthétique des représentations); bien qu'il me faille encore veiller à ne pas confisquer la parole aux sujets concernés et à ne pas ignorer la «science des opprimé.e.s» (pour reprendre la formule de Wittig), c'est-à-dire l'expertise des agents concernés. Je pense qu'il y a divers degrés d'investissement politique personnel dans les subcultures sexuelles : on peut considérer qu'un.e hétérosexuel.le qui finit par adopter les pratiques culturelles (et, plus particulièrement, sexuelles) d'un autre groupe sexuel n'est pas "post-straight»; il ou elle n'est tout simplement plus hétérosexuel.le et appartient désormais à ce nouveau groupe « d'adoption » (je ne sais pas s'il s'agit $\mathrm{du}$ bon terme). C'est une autre démarche, un autre type d'engagement, cela me paraît tout à fait envisageable et il ne s'agira pas, dans ce cas précis, d'une appropriation culturelle, on pourrait plutôt parler d'une sorte de transition culturelle. 
12 Pour autant, la pensée straight a déjà imprimé mon corps hétérosexuel, c'est un fait contre lequel je ne vois pas encore comment lutter, mais je peux tout de même, malgré ce corps hégémonique, cesser de participer à l'inoculation sociale, politique et scientifique du langage dominant de l'hétérosexualité de bien d'autres manières. C'est ainsi que, partant de l'expérience d'un corps qui est certes hégémonique, mais qui a été informé par la critique queer, crip, féministe et afroféministe, je produis un langage social et scientifique en accord avec l'esprit de la contestation wittigienne, et je cherche à inventer et à introduire - en société et dans les sciences - un mode de pensée que je qualifierais de post-straight.

GLAD!: Pouvez-vous développer ce que vous entendez par langage social ? Est-ce que cela signifie que vous usez d'une façon particulière des savoirs situés, par exemple, dans vos écrits?

Je dis « langage social » pour exprimer mes « relations signifiantes avec autrui ». C'est effectivement ce qui caractérise le mieux la spécificité de ma position politique personnelle en tant qu'homme hétérosexuel post-straight. Mon intention politique est de savoir gérer mon attitude sociale avec les autres, de prendre conscience de mes paroles et de mes actes en ce qu'ils peuvent générer en terme de relation de pouvoir asymétrique. Du point de vue scientifique, cela engage une attitude double, ou en deux temps: en tant que théoricien de l'art, je suis intéressé et sensible aux productions artistiques des artistes minoritaires, parce qu'elles me semblent œuvrer dans une perspective politique (d'utilité publique) qui correspond à ma propre conception esthétique de l'art. Bien entendu, je sais pertinemment que je ne peux pas totalement m'identifier à l'ensemble des travaux artistiques minoritaires que j'étudie, je dois donc faire usage de la «science des opprimé.e.s ", pour appréhender certaines œuvres. Cela me permet d'adopter un point de vue situé, bien sûr, mais qui est quelque peu aiguillé par le regard minoritaire des spécialistes qui parlent en tant qu'agents concerné.e.s. Je parle ainsi d'une attitude scientifique double car, oui, je regarde et j'analyse les représentations à partir de mon propre point de vue, mais d'un autre côté, ce point de vue est fortement informé par les discours minoritaires.

Cela me permet de différencier l'hétérosexualité post-straight de l'hétérosexualité straight, sans toutefois me dédouaner de ma situation politique, en tant qu'homme hétérosexuel blanc, vis-à-vis de la domination institutionnelle et sociale des minorités. Malgré les apparences de binarité que peut revêtir cet appareil conceptuel, il ne s'agit pas d'opposer deux hétérosexualités de manière dualiste, mais simplement de différencier deux types de subjectivités hétérosexuelles, et il en est bien d'autres ${ }^{22}$.

Lorsque je m'identifie comme post-straight, je m'auto-définis comme un sujet d'énonciation hétérosexuel qui ne se considère pas plus naturel ou normal que les autres, mais qui est plutôt une subjectivité possible (parmi tant d'autres). Malheureusement, le pouvoir social m'érige parfois encore comme un sujet porteur de la domination, mais c'est une gratification que je rejette fondamentalement par ma 
posture contestataire de sujet post-straight. L'hétéro post-straight que je suis réfléchit à comment s'allier aux groupes minoritaires pour plus de justice sociale et pour une conception du monde ouverte à toute forme (librement consentie) de subjectivités, de corporéités et de sexualités. Mon engagement post-straight est en constante redéfinition, c'est une réflexion en cours, mais il implique un rapport au monde qui, s'il n'est évidemment pas queer (puisqu'il ne subit pas l'oppression sexiste, homophobe et raciste), n'est pas straight pour autant. Ma subjectivité post-straight m'invite bien souvent à entrer dans un conflit intellectuel avec mon propre milieu et ma propre communauté (sociale et scientifique) sur les questions identitaires et/ou sexuelles. Mais bien que j'aie conscience des enjeux politiques, psychologiques et anthropologiques de l'hétérosexualité, je reste investi dans des relations affectives avec des personnes dites de « sexe différent »- en l'occurrence une seule, puisque je suis engagé dans un couple monogame. Je ne cherche pas à imiter ou à m'approprier les cultures minoritaires, mais juste à inventer un "vivre avec", ainsi qu'à développer les modalités d'une alliance possible dans la contestation de la pensée straight - la production de théories critiques en est une, c'est en tout cas ma conviction, même si l'on pourra me reprocher un engagement pour l'instant trop théorique et pas assez social (mais cela évoluera sans doute avec le temps par l'invention future de modes d'actions aujourd'hui inexistants). Depuis plusieurs années maintenant, ma vie est étrangement affiliée à des questions de genres, de races, de classes, de sexualités et d'identités qui m'interpellent, et ce, malgré ma situation sociale qui a le privilège de n'être jamais remise en question par le pouvoir straight. J'ignore si d'autres hétéros se reconnaissent dans cette posture que je nomme post-straight - oserais-je un jour employer un "nous" de solidarité? J'ignore également dans quelle mesure des hétéros post-straights (en devenir) pourront être utiles aux minorités contestataires, mais gageons de ne pas devenir des alliée.es encombrant.e.s. En tout état de cause, il me faut rester ouvert à toute discussion critique autour de cette invention du sujet post-straight, dans l'espace social comme dans l'espace scientifique.

GLAD! : Autour de la question de l'identité, du sujet intentionnel, en regard des rapports de pouvoirs dans lequel le sujet est engagé : être post-straight est-il une histoire de décision individuelle? Comment rendre lisible/visible le post-straight? Y a-t-il une agentivité post-straight, une culture poststraight? Comment le post-straight se situe-t-il dans le "système social » vis-à-vis des queers mais aussi des hétéros straights? Quels rapports de pouvoirs complexes sont joués et déjoués?

Alban Jacquemart a écrit un ouvrage d'histoire sociale sur Les Hommes dans les mouvements féministes ${ }^{23}$ qu'il décrit, ironiquement bien sûr, comme un "engagement improbable». Ce livre témoigne d'une volonté chez certains hommes de combattre leur propre domination, et il me semble que le scénario se répète pour les hétérosexuel.le.s qui souhaitent déjouer la pensée straight critiquée par la théorie queer ou encore les Blanc.he.s qui s'investissent dans la lutte antiraciste et les mouvements décoloniaux. Ce sont ceux.celles-là que je qualifie de post-straight. Je pense avoir mis en évidence que cette forme d'engagement, dont j'ai encore du mal à définir les contours, résulte d'une construction sociale de la subjectivité qui dépasse sa propre individualité, du fait d'une sensibilité particulière pour la 
situation précaire d'autrui. Mais peut-être qu'il apparaîtra, ou même qu'il existe déjà, des discours dissonants qui me contredisent ou contrediront ; cette réflexion auto-théorique est loin d'être close.

Le post-straight est une invention conceptuelle de ma part, il n'y a donc pas de culture post-straight à proprement parler, mais il a existé et il existe toujours des rassemblements et des groupes de réflexion sur la possibilité pour un homme d'être féministe. Je ne suis pas en mesure, aujourd'hui, d'en discuter de manière approfondie pour évoquer l'éventualité d'un mouvement en construction et d'un contenu politique qui nous éclairerait effectivement sur les rapports de pouvoirs complexes qui sont joués et déjoués au sein de ces micropolitiques. Néanmoins, ces évènements philosophiques et culturels ont le mérite d'exister, témoignant d'une agentivité et d'un désir collectivement partagé (par certains) de transcender le « devenir oppresseur » de la masculinité.

\section{BIBLIOGRAPHIE}

BARRÉ, Alain (éd.). 1995. Musique et politique : les répertoires de l'identité. Rennes : Presses universitaires de Rennes

BOISVERT, Jacques. 1999. La Formation de la pensée critique. Théorie et pratique. Bruxelles : Éditions De Boeck Université

BOURCIER, Marie-Hélène/Sam. 2015. Queer Zone 3 : identités, cultures, politiques. Paris : Amsterdam. BUTLER, Judith. 2009 [1993]. Ces Corps qui comptent : de la matérialité et des limites discursives du sexe (trad. C. Nordmann). Paris : Éditions Amsterdam.

BUTLER, Judith. 2005 [1990]. Trouble dans le genre : pour un féminisme de la subversion (trad. C. Kraus). Paris : Éditions La Découverte.

CRENSHAW, Kimberlé Williams. 2005 [1994]. « Cartographies des Marges : intersectionnalité, politique de l'identité et violences contre les femmes de couleur » (trad. O. Bonis) Cahiers du Genre [En ligne], 2 (39), consulté le 19 décembre. URL : https://www.cairn.info/revue-cahiers-dugenre-2005-2-page-51.htm

DESPENTES, Virginie. 2017. « Les mecs sont extrêmement lents à s'emparer de la question de la masculinité » Les Inrocks [En ligne], consulté le 10 septembre 2017. URL : http:// www.lesinrocks.com/2016/10/23/actualite/medias-actualite/virginie-despentes-mecsextremement-lents-a-semparer-de-question-de-masculinite-11874131/

JACQUEMART, Alban. 2015. Les hommes dans les mouvements féministes. Sociohistoire d'un engagement improbable. Rennes : Presses universitaires de Rennes.

PRECIADO, Paul B. 2017. « Le prix de votre normalité sexuelle est notre "intersexualicide" » Libération, consulté le 13 septembre 2017. URL : http://www.liberation.fr/debats/2017/06/02/leprix-de-votre-normalite-sexuelle-est-notre-intersexualicide_1574196 
WITTIG, Monique. 2007 [1978]. La Pensée straight. Paris : Éditions Amsterdam.

\section{NOTES}

1. WITTIG, Monique. 2007 [1978]. La Pensée straight. Paris : Éditions Amsterdam, p. 53-61.

2. Id., p. 57-58.

3. Wittig cite notamment Lacan, elle fait également mention de l'industrie pornographique.

4. Cf. http://www.gouvernement.fr/action/le-mariage-pour-tous

5. Voir notamment BOURCIER, Marie-Hélène/Sam. 2015. Queer Zone 3 : identités, cultures, politiques. Paris : Amsterdam, p. 281-292.

6. Bien entendu, rien n'oblige les homosexuel.le.s à se marier, mais l'entrée en vigueur de cette loi est clairement incitative (du fait des avantages sociaux qui accompagnent le mariage). Elle crée par ailleurs un clivage intracommunautaire à travers un phénomène d'homonormativité qui tente de dévaluer les subcultures sexuelles queer d'une manière similaire à la pensée straight. Cf. Bourcier, op. cit.

7. WITTIG. op. cit., p. 58-59.

8. BUTLER, Judith. 2009 [1993]. Ces Corps qui comptent: de la matérialité et des limites discursives du sexe (trad. C. Nordmann). Paris : Amsterdam.

9. WITTIG, op. cit., p. 59.

10. PRECIADO, Paul B. 2017. "Le prix de votre normalité sexuelle est notre "intersexualicide" " Libération, consulté le 13 septembre 2017. URL : http://www.liberation.fr/debats/2017/06/02/leprix-de-votre-normalite-sexuelle-est-notre-intersexualicide_1574196

11. BUTLER, Judith. 2005 [1990]. Trouble dans le genre : pour un féminisme de la subversion (trad. C. Kraus). Paris : La Découverte.

12. Que le/la lecteur.trice n'y voie aucun élitisme de ma part, mais au contraire une forme d'humilité et de gratitude. Sans ces études je ne pourrais pas être en train de théoriser comme je suis en train de le faire. Par ailleurs, il me semble important de signaler, pour établir le cadre d'une réflexivité objective, que j'ai été formé par des enseignant.e.s marxistes, féministes et queer au cours de ma formation universitaire. Je suis actuellement titulaire d'un doctorat en arts spécialisé dans l'étude des influences des études minoritaires dans la création plasticienne contemporaine (la performance en particulier).

13. Cette question a déjà été abordée par la sociohistoire. Cf. JACQUEMART, Alban. 2015. Les hommes dans les mouvements féministes. Sociohistoire d'un engagement improbable. Rennes : Presses universitaires de Rennes.

14. BUTLER. Trouble..., op. cit.

15. WACHOWSKI, Lana \& WACHOWSKI, Lily. 1999. Matrix. New York : Warner Bros.

16. BOISVERT, Jacques. 1999. La Formation de la pensée critique. Théorie et pratique. Bruxelles: Éditions De Boeck Université, p. 27.

17. Notamment à cause de l'institutionnalisation et de la verticalité de l'apprentissage de la critique qu'implique cette conception.

18. Il s'agit toutefois d'un fait avéré qui peut être généralisé, les arts participent au développement de l'esprit critique des individu.e.s, par le biais d'une construction subjective qui sera orientée par la dialectique politique et culturelle des œuvres et des artistes qu'il apprécie. Voir par exemple BARRE, Alain (éd.). 1995. Musique et politique : les répertoires de l'identité. Rennes : Presses universitaires de Rennes.

19. CRENSHAW, Kimberlé Williams. 2005 [1994]. «Cartographies des Marges : intersectionnalité, politique de l'identité et violences contre les femmes de couleur » (trad. O. Bonis) Cahiers du Genre 
[En ligne], 2 (39), consulté le 19 décembre. URL: https://www.cairn.info/revue-cahiers-dugenre-2005-2-page-51.htm

20. Comme le fait de me teindre les cheveux en rose afin d'arborer en tant que garçon une couleur socialement considérée comme féminine et cultiver une apparence queercore alors même que j'étais un homme hétérosexuel cisgenre.

21. Par exemple : DESPENTES, Virginie. 2017. « Les mecs sont extrêmement lents à s'emparer de la question de la masculinité » Les Inrocks [En ligne], consulté le 10 septembre 2017. URL : http:// www.lesinrocks.com/2016/10/23/actualite/medias-actualite/virginie-despentes-mecs-

extremement-lents-a-semparer-de-question-de-masculinite-11874131/

22. Comme je l'ai dit auparavant, il existe encore d'autres hétérosexualités minoritaires comme l'hétérosexualité racisée, queer ou trans, que je n'aborderai pas dans ce texte, mais qui se différencient également de l'hétérosexualité straight.

23. JACQUEMART, op. cit.

\section{RÉSUMÉS}

Cette expérience d'écriture s'inscrit dans une démarche artistique - à la fois personnelle et scientifique -, questionnant la pratique auto-ethnographique comme une forme plastique. Elle résulte d'une réflexion esthétique sur la possibilité d'une philosophie de soi - donner un « récit de soi », pour reprendre la formule de Judith Butler - comme travail artistique conceptuel. S'inscrivant dans la perspective foucaldienne et butlérienne selon laquelle le corps est sculpté par les discours, mon travail propose ainsi d'interroger la capacité d'un corps à pouvoir se dire et se dédire, et donc travailler sa forme (sociale) par l'écriture de soi; et dans le cadre plus particulier de ce texte, il s'agira d'éprouver mon corps dans sa faculté de pouvoir dire et dédire sa propre hétérosexualité.

This writing experience is part of an artistic process-both personal and scientific-questioning self-ethnographic practice as a creative form. It leads to an aesthetic reflection on the possibility of making a philosophy about the self-“Giving an Account of Oneself”, to use the Judith Butler's formula- as a conceptual artistic work. As part of the Foucauldian and Butlerian perspective according to which the body is sculpted by discourses, my work proposes to question a body's capacity to say and to withdraw from oneself, and thus to work its (social) form by the selfwriting; in the particular context of this text, I wanted to try my body's ability to say and withdraw (from) its own heterosexuality.

\section{INDEX}

Thèmes : Explorations

Keywords : auto-ethnography, art, feminism, sexuality, class

Mots-clés : auto-ethnographie, art, féminisme, sexualité, classe 


\section{AUTEUR}

\section{LUC SCHICHARIN}

CREM (Centre de recherche sur les médiations)

Luc Schicharin est docteur en arts, esthétique et science de l'art. Sa thèse de doctorat s'intitule 'L'esthétique drag, de la performance travestie à l'art transgenre. Usages et contre-usages de la théorie butlérienne ». Il est actuellement chargé de cours en arts plastiques à l'Université de Lorraine, campus de Metz. 